\title{
NÍVEL DE DOR, ANSIEDADE E DEPRESSÃO EM PACIENTES COM DOR LOMBAR ATENDIDOS EM UM CENTRO DE FISIOTERAPIA
}

Silas de Oliveira Damasceno, José Henrique Piedade Cardoso, Raíssa Puzzi Ladvig, Luana Martins de Paula, Renilton José Pizzol, Ana Lúcia de Jesus Almeida

Universidade Estadual Paulista "Júlio Mesquita Filho" - UNESP, Departamento de Fisioterapia, Curso de Fisioterapia, Presidente Prudente, SP. E-mail: silas.damasceno10@hotmail.com

\section{RESUMO}

A dor, a ansiedade e a depressão são sintomas que necessitam de atenção na prática fisioterapêutica, uma vez que estas influenciam na funcionalidade dos pacientes, assim, entendelas se torna ferramenta importante. O estudo tem por objetivo verificar os níveis de dor, depressão e ansiedade em pacientes atendidos em um centro de fisioterapia. Estudo clínico transversal, na qual foi aplicado um questionário sociodemográfico, a Escala Visual Analógica (EVA), a Escala Hospitalar de Ansiedade e Depressão (HAD) e o Questionário de Roland-Morris (RM). Sete pacientes participaram e apresentaram pontuação de RM de 18,57 $\pm 2,63$ pontos, HAD no tópico ansiedade $12,71 \pm 5,43$ pontos, HAD depressão $9,57 \pm 5,22$ pontos e a EVA em repouso apresentou média de 4,42 $\pm 3,9$ pontos de dor e em movimento evidenciou $8,71 \pm 0,95$. Conclui-se que a população com dor lombar analisada apresenta alta intensidade de dor, provável ansiedade e possível nível de depressão.

Palavras-chave: Dor lombar, ansiedade, depressão, fisioterapia.

\section{LEVEL OF PAIN, ANXIETY AND DEPRESSION IN PATIENTS WITH LOW BACK PAIN TREATED IN A PHYSIOTHERAPY CENTER}

\begin{abstract}
Pain, anxiety, and depression are symptoms that need attention in physiotherapeutic practice, once they influence the functionality of patients, so understanding them becomes an important tool. The aim of the study was to verify the levels of pain, depression, and anxiety in patients attended at a physiotherapy center. A cross-sectional clinical study in which a sociodemographic questionnaire was applied, the Visual Analogue Scale (VAS), the Hospital Anxiety and Depression Scale (HADS) and the Roland Morris Disability Questionnaire (MRQ). Seven patients participated and presented a MRI score of $18.57 \pm 2.63$ points, HADS in the topic anxiety $12.71 \pm 5.43$ points, HADS depression $9.57 \pm 5.22$ points and the resting VAS presented an average of $4,42 \pm 3.9$ pain points and in motion showed $8.71 \pm 0.95$. It is concluded that the population with low back pain has high intensity of pain, probable anxiety and possible level of depression.
\end{abstract}

Keywords: Low back pain, anxiety, depression, physiotherapy. 


\section{INTRODUÇÃO}

A dor é um episódio com múltiplas dimensões, assim os pacientes devem ser tratados de acordo com suas especificidades. O fisioterapeuta deve realizar uma ampla avaliação abordando os aspectos espirituais, psíquicos, físicos e sociais ${ }^{1}$.

Para isso, o terapeuta tem que ter em mente que a dor é um fator que prejudica a qualidade de vida, pois o adoecimento não acomete apenas o físico, mas a vida como um todo de maneira biopsicossocial ${ }^{2}$.

A dor é descrita como o quinto sinal vital e deve ser registrada ao mesmo tempo, ambiente clínico, em que são analisados os sinais vitais, pulso, respiração, pressão arterial e temperatura, como indicado pela Agência Americana de Pesquisa e Qualidade em Saúde Pública e a Sociedade Americana de Dor ${ }^{3}$.

A dor lombar tem sua ocorrência por trauma, lesão mecânica, lesões medulares, infecções, inflamações e outras causas, tendo apresentado incidência em algum período da vida de boa parte dos adultos ${ }^{4}$.

Quando em altos níveis a dor lombar pode gerar incapacidades levando a restrições de certas atividades, impedindo o individuo de realizar funções laborais, atividades de lazer e alterando o convívio familiar ${ }^{4,5,6}$. Esses problemas tem alta prevalência no mundo e no Brasil, levando a custos econômicos imensos, estando estes gastos intimamente ligados às disfunções emocionais, desesperança, ansiedade e depressão ${ }^{7,8,9,10}$.

A ansiedade e a depressão são problemas muito frequentes em pacientes com dor lombar, existindo na literatura evidências que relaciona estas com altos níveis de dor lombar, assim olhar com mais critério para esses aspectos surte como forma de intervenção para propor tratamentos cada vez mais direcionado aos pacientes que necessitam de atendimento fisioterapêutico ${ }^{11,12}$.

Devido às circunstâncias citadas anteriormente, o presente estudo foi feito com a proposição de contribuir cientificamente para um maior entendimento da dor lombar, depressão e ansiedade, diante disso, o objetivo foi verificar os níveis de dor, depressão e ansiedade em pacientes com dor lombar atendidos em um centro de fisioterapia.

\section{METODOLOGIA}

Estudo clínico transversal com participação de pacientes atendidos em um centro de referência em Fisioterapia (CRF) de Presidente Prudente/SP.

Foram incluídos pacientes maiores de idade, de ambos os sexos, com relato inicial de qualquer sintomatologia de dor lombar, todavia, foi estabelecida nota de corte de 14 pontos para a pontuação da escala de Roland-Morris, ausência de déficits cognitivos ou físicos que impedissem a realização do estudo e que anuíram ao Termo de Consentimento Livre e Esclarecido, aprovado pelo Comitê de Ética em Pesquisa da FCT/UNESP campus de Presidente Prudente/SP sob o parecer de número 41034914.1.0000.5402.

Foram aplicados questionários sociodemográficos para caracterização da população, o questionário de Roland-Morris de Incapacidade, a Escala de Ansiedade e Depressão (HAD) e a Escala Visual Analógica.

O questionário de Roland-Morris mede a incapacidade de pessoas com dor lombar crônica e é validade no Brasil. Possui 24 afirmações, nas quais o paciente deve apontar por meio de "sim" consentindo a afirmação ou "não" refutando a afirmação. A pontuação varia de 0 a 24 pontos, sendo os dois extremos, respectivamente, sem incapacidade funcional e incapacidade funcional total, entretanto, pontuações acima de 14 pontos indicam uma incapacidade significativa ${ }^{13}$.

A ansiedade e depressão foram avaliadas pela HAD que compõe 14 afirmativas com quatro opções de respostas por item e que deverá ser respondida, por exemplo, por meio de "a maior parte do tempo" 3 pontos, "boa parte do tempo" 2 pontos, "de vez em quando" 1 ponto ou "nunca" 0 ponto. A escala subdivide as questões referentes a ansiedade nas questões ímpares e as 
referentes a depressão nas questões pares. A pontuação de 0 a 7 pontos indica improvável, 8 a 11 pontos uma situação possível (questionável ou duvidosa) e 12 a 21 pontos mostra uma provável ocorrência de depressão e/ou ansiedade na população analisada ${ }^{14}$.

A Escala Visual Analógica foi utilizada para verificar a intensidade de dor referida pelo paciente, que possui uma pontuação de 0 a 10, sendo de 0 a 2 indica um nível de dor leve, de 3 a 7 pontos corresponde ao nível moderado de dor e de 8 a 10 pontos o nível intenso de algum processo álgico ${ }^{15}$.

Para análise dos dados foi utilizado o Microsoft Excel ${ }^{\circledR}$, utilizando-se de dados descritivos com média, desvio padrão e porcentagem.

\section{RESULTADOS}

Foram encontrados 12 pacientes, todavia, apenas 7 pacientes participaram efetivamente desde estudo, sendo seis mulheres e um homem com média de idade geral de $58 \pm 14,3$ anos, ao estado civil $71,41 \%$ eram casadas, $14,28 \%$ solteiros e $14,28 \%$ divorciadas, além de $57,12 \%$ apresentaram hipertensão arterial sistêmica como outra comorbidade.

A pontuação de Roland-Morris apresentou 18,57 $\pm 2,63$ pontos, HAD no tópico ansiedade mostrou 12,71 $\pm 5,43$ pontos e HAD depressão 9,57 $\pm 5,22$ pontos e a EVA em repouso apresentou média de 4,42 $\pm 3,9$ grau de dor e em movimento evidenciou 8,71 $\pm 0,95$ pontos de dor.

\section{DISCUSSÃO}

No presente estudo observou-se que os pacientes apresentaram uma incapacidade significativa referente à dor lombar, foi observado também, uma provável situação de ansiedade e uma possível depressão, além de alta intensidade de dor lombar verificado pelos escores das ferramentas aplicadas na avaliação.

A dor é um processo que influencia no desenvolvimento das atividades do cotidiano ${ }^{2}$. Observou-se que o quadro álgico em movimento é reportado por todos os pacientes em um nível de oito ou mais na EVA, enquanto em repouso, a dor fica em um nível moderado, demonstrando que os pacientes realizam um repouso indevido frente à condição dolorosa para evitá-la, todavia, tal fato se torna ainda mais prejudicial para funcionalidade e qualidade de vida dos indivíduos ${ }^{15}$.

As mulheres apresentaram maior nível de incapacidade devido à dor lombar do que os homens e também apresentaram estado de saúde mais comprometido, tendo a hipertensão arterial sistêmica como comprometimento mais frequente, dados também observados em outro estudo ${ }^{16}$.

A ansiedade e a depressão apresentaram dados importantes que remontam para aspectos de saúde pública, pois se encontrou níveis altos por meio da HAD que podem influenciar em risco de mortes, formação de doenças cancerígenas, doenças coronarianas, dentre outras e estabelecidos na literatura ${ }^{17}$.

Outros estudos acharam grandes correlações entre a ansiedade, depressão e a dor lombar e apontaram restrições nas atividades de vida diária, como deambular, realizar compras, carregar objetos, visitar familiares e outros $7,8,9,17$.

Aparentemente, a dor lombar e a depressão levam a duas premissas ${ }^{18,19}$, a primeira é que os sintomas depressivos podem gerar riscos para o desenvolvimento de uma futura dor $\operatorname{lombar}^{20,21}$, e a segunda, que a depressão seja desenvolvida após a Dor lombar ${ }^{22,17,19}$. Apesar de não existir um consenso entre qual acometimento ocorre primeiro, existe concordância que estes são grandes acometimentos encontrados entre as diversas disfunções corporais na prática clínica ${ }^{23}$.

Embora da contribuição que o estudo trouxe, ainda assim, apresentam algumas limitações, nas quais se referem à amostra pequena, idades diferentes, diferentes sintomatologias e classificações do quadro álgico de coluna lombar distintas, o que podem ter influenciado na 
composição do resultado final, assim, devem-se levar em consideração esses tópicos levantados para resultados mais precisos.

Conclui-se que a população com dor lombar analisada apresenta alta intensidade de dor, provável ansiedade e possível nível de depressão, o que afeta sobremaneira a qualidade de vida destes pacientes e influência diretamente na prática fisioterapêutica, diante disso, explorar esta temática se torna fundamental.

\section{DECLARAÇÃO DE CONFLITO DE INTERESSE}

Os autores declaram não haver qualquer potencial conflito de interesse que possa interferir na imparcialidade deste trabalho científico.

\section{REFERÊNCIAS}

1. Hortense P, Sousa FAEF. Escalonamento comparativo de diferentes dores nociceptivas e neuropáticas por meio de métodos psicofísicos variados. Rev. Latino-Am. Enfermagem. 2009; 17(2):207-14. https://doi.org/10.1590/S0104-11692009000200011

2. Sanches LM, Boemer MR. O convívio com a dor: um enfoque existencial. Rev Esc Enferm USP. 2002; 36(4):386-93. DOI: http://dx.doi.org/10.1590/S0080-62342002000400013.

3. Sousa FAEF. Dor: o quinto sinal vital. Rev. Latino-Am. Enfermagem. 2002; 10(2):446-7. DOI: http://dx.doi.org/10.1590/S0104-11692002000300020.

4. Brito SJ, Lima MM, Silva ASB, Macêdo MCA. Prevalência de lombalgias musculoesqueléticas dependentes em comerciários. S A N A R E, ISSNe:2317-7748, V.14 - Suplemento 1 - COPISP - 2015.

5. Asghari A. Psychometric properties of a modified version of the Roland Morris disability questionnaire (M-RMDQ). Arch Iran Med. 2011; 14(5):327-31. DOI: http://dx.doi.org/007.

6. Meucci RD, Fassa AG, Paniz VMV, Silva MC, Wegman DH. Increase of chronic low back pain prevalence in a medium-sized city of southern Brazil. BMC Musculoskel Disord. 2013; 14:155-61. DOI: http://dx.doi.org/10.1186/1471-2474-14-155.

7. Salvetti MG, Pimenta CAM, Braga PE, Corrêa CF. Incapacidade relacionada à dor lombar crônica: prevalência e fatores associados. Rev Esc Enferm USP. 2012; 46(Esp):16-23. DOI: http://dx.doi.org/10.1590/S0080-62342012000700003.

8. Stefane T, Santos AM, Marinovic A, Hortense P. Chronic low back pain: pain intensity, disability and quality of life. Acta Paul Enferm. 2013; 26(1):14-20. DOI: http://dx.doi.org/10.1590/S010321002013000100004 .

9. Amaral V, Marchi L, Oliveira L, Pimenta L. Prevalência e relação de fatores emocionais e clínicos em pacientes com discopatia degenerativa. Coluna/Columna. 2010; 9(2):150-6. DOI: http://dx.doi.org/10.1590/S1808-18512010000200011.

10. Matsudaira K, Konishi H, Miyoshi K, Isomura T, Inuzuka K. Potential Risk Factors of Persistent Low Back Pain Developing from Mild Low Back Pain in Urban Japanese Workers. Plos One. 2014; 9(4):e93924. DOI: http://dx.doi.org/10.1371/journal.pone.0093924. 
11. Castro MMC, Daltro C. Sleep patterns and symptoms of anxiety and depression in patients with chronic pain. Arq Neurol. 2009; 67(1):25-8. DOI: http://dx.doi.org/10.1590/S0004282X2009000100007.

12. Preuper HRS, Reneman MF, Boonstra AM, Dijkstra PU, Versteegen GJ, Geertzen JH, et al. Relationship between psychological factors and performance-based and selfreported disability in chronic low back pain. Eur Spine. 2008; 17(11):1448-56. DOI: http://dx.doi.org/10.1007/s00586$\underline{008-0772-0 .}$.

13. Nusbaum L, Natour J, Ferraz MB, Goldenberg J. Translation, adaptation and validation of the RolandMorris questionnaire - Brazil Roland-Morris. Braz J Med Biol Res. 2001; 34(2):203-10. DOI: http://dx.doi.org/10.1590/S0100-879X2001000200007.

14. Zigmond AS e Snaith RP. The Hospital Anxiety and Depression Scale. Acta Psychiatrica Scandinavica. 1983; 67, 361-370. https://doi.org/10.1111/j.1600-0447.1983.tb09716.x

15. Martinez JE, Grassi DC, Marques LG. Análise da aplicabilidade de três instrumentos de avaliação de dor em distintas unidades de atendimento: ambulatório, enfermaria e urgência. Ver Bras Reumatol. 2011; 51(4):299-308. DOI: http://dx.doi.org/10.1590/S0482-50042011000400002.

16. Malta DC, Oliveira MM, Andrade SSCA, Caiaffa WT, Souza MFM, Bernal RTI. Fatores associados à dor crônica na coluna em adultos no Brasil. Rev Saude Publica. 2017; 51 Supl 1:9s. DOI: http://dx.doi.org/10.1590/s1518-8787.2017051000052.

17. Andrade, LH, Baptista MC, Alonso J, Petukhova M, Bruffaerts R, Kessler, et al. Days outof-role due to common physical and mental health problems: Results from the Sao Paulo Megacity Mental Health Survey, Brazil. Clinics (Sao Paulo). 2013; 68(11): 1392-1399. DOI: 10.6061/clinics/2013(11)02.

18. Instituto Brasileiro de Geografia e Estatística [IBGE]. Perfil dos idosos responsáveis pelo domicílio 2000. Rio de Janeiro: IBGE; 2002.

19. Reid MC, Williams CS, Concato J, Tinetti ME, Gill TM. Depressive symptoms as a risk factor for disabling back pain in community-dwelling older persons. J Am Geriatr Soc. 2003; 51(12):17172003. DOI: https://doi.org/10.1046/i.1532-5415.2003.51554.x.

20. Andersson GB. Epidemiological features of chronic low-back pain. Lancet. 1999; 354(9178):581-5. DOI: https://doi.org/10.1016/S0140-6736(99)01312-4.

21. Hartvigsen J, Frederiksen H, Christensen K. Physical and mental function and incident low back pain in senior. A population-based two prospective study of 1387 twins age 70 to 100 years. Spine. 2006; 31(14):1628-32. DOI: https://doi.org/10.1097/01.brs.0000222021.00531.ea.

22. Meyer T, Cooper J, Raspe H. Disabling low back pain and depressive symptoms in the community-dwelling elderly. Spine. 2007; 32(21):2380-6. DOI: https://doi.org/10.1097/BRS.0b013e3181557955. 
23. Kaptan $\mathrm{H}$, Yalçin ES, Kasimcan O. Correlation of low back pain caused by lumbar spinal stenosis and depression in women: a clinical study. Arch Orthop Trauma Surg. 2012; 132(7):963-7. DOI: https://doi.org/ 10.1007/s00402-012-1513-8. 